\title{
Estrutura e síndromes de dispersão de espécies arbóreas em um trecho de mata ciliar, Sirinhaém, Pernambuco, Brasil
}

\author{
Roseane Karla Soares da Silva¹, Ana Lícia Patriota Feliciano¹, Luiz Carlos Marangon¹, Rosival Barros de Andrade Lima¹, \\ Wedson Batista dos Santos ${ }^{1}$ \\ ${ }^{1}$ Universidade Federal Rural de Pernambuco, Rua Dom Manoel de Medeiros, s/n, Dois Irmãos, CEP 52171-900, Recife, PE, Brasil
}

"Autor correspondente:

aneufrpe@ig.com.br

Termos para indexação:

Dispersão de sementes

Mata Atlântica

Fitossociologia

Index terms:

Seed dispersal

Atlantic Forest

Phytosociology

\section{Histórico do artigo:}

Recebido em 29 maio 2011

Aprovado em 06 dez 2011

Publicado em 30 mar 2012

doi: 10.4336/2012.pfb.32.69.01
Resumo - Este trabalho teve por objetivo conhecer a fitossociologia do componente arbóreo em uma mata ciliar de um córrego que deságua no Rio Sirinhaém, localizado no Engenho Buranhém, Sirinhaém, PE e identificar as síndromes de dispersão das espécies. O córrego está situado em um fragmento de Floresta Ombrófila Densa de Terras Baixas, com 272 ha. Utilizou-se como área amostral um hectare (40 unidades amostrais, $10 \mathrm{~m} \times 25 \mathrm{~m}$ ) onde foram registrados 1.307 indivíduos arbóreos com circunferência à altura do peito $(\mathrm{CAP}) \geq 15 \mathrm{~cm}$. Foram identificadas 118 espécies, pertencentes a 40 famílias botânicas. Protium heptaphyllum e Pouteria sp.1 foram as espécies mais abundantes. Em termos de valor de importância (VI), as espécies Protium heptaphyllum, Pouteria sp.1 e Virola gardneri estão entre as mais importantes ecologicamente. As síndromes de dispersão predominantes foram: zoocoria $(72,8 \%)$, autocoria $(13,6 \%)$ e anemocoria (4,8\%). Não foi possível determinar a síndrome de dispersão de $8,8 \%$ das espécies estudadas.

\section{Structure and dispersal syndromes of tree species in a stretch of riparian vegetation, Sirinhaém, Pernambuco State, Brazil}

\begin{abstract}
This study aimed to know the phytosociology of the tree component of riparian vegetation in a stream that flows into the Sirinhaém River in Engenho Buranhém, Sirinhaém, Pernambuco State, and identify the species dispersal syndromes. The stream is located in a fragment of dense rain forest of the lowlands, with $272 \mathrm{ha}$. It was sempled used one hectare ( 40 sampling units of $10 \mathrm{~m}$ x $25 \mathrm{~m}$ each). It was registered 1,307 trees with circumference at breast height $(\mathrm{CAP}) \geq 15 \mathrm{~cm}$. We identified 118 species belonging to 40 botanical families. Protium heptaphyllum and Pouteria sp. 1 were the most abundant species. In terms of importance value (IV), Protium heptaphyllum, Pouteria sp.1 and Virola gardneri are among the most important ecologically species. The predominant dispersal syndromes were zoocory (72.8\%), autocory (13.6\%) and anemochory (4.8\%). It was not possible to determine the type of dispersal of $8.8 \%$ of the species studied.
\end{abstract}

\section{Introdução}

A Mata Atlântica é a segunda maior floresta pluvial tropical do continente americano, que originalmente se estendia de forma contínua ao longo da costa brasileira, penetrando até o leste do Paraguai e nordeste da Argentina em sua porção sul (Galindo-Leal \& Câmara, 2005).

Os poucos núcleos que ainda podem ser caracterizados como florestas primárias estão concentrados em áreas de altitude elevada e acesso difícil, onde a prática agrícola 
ou madeireira se torna inviável, além de outras poucas áreas de preservação ambiental (Câmara, 2003; Moreno et al., 2003). De modo geral, os remanescentes de Floresta Atlântica encontram-se em estágio de sucessão secundária, fragmentados, alterados e empobrecidos em sua composição florística original. Porém, esses fragmentos florestais nativos são valiosos recursos naturais renováveis, passíveis de utilização pelas gerações presentes e futuras (Souza et al., 2002).

Nas últimas décadas, houve considerável avanço nos estudos de comunidades florestais, principalmente por causa de sua importância para a conservação da diversidade biológica. Essa importância se torna cada dia mais acentuada, devido ao processo desordenado de ocupação do solo que ainda persiste nas mais diversas regiões do país, transformando formações florestais contínuas em fragmentos (Santos \& Vieira, 2006 ; Carvalho et al., 2007).

As matas ciliares não escaparam da destruição e foram alvo de todo tipo de degradação, sendo notório que muitas cidades brasileiras foram formadas às margens dos rios (Ferreira \& Dias, 2004). A preservação e a recuperação das matas ciliares e o manejo sustentável de bacias hidrográficas são de extrema importância para a manutenção dos ecossistemas aquáticos, pois auxiliam na infiltração de água no solo, facilitando o abastecimento do lençol freático; mantêm a qualidade da água, dificultando o escoamento superficial de partículas e sedimentos, que causam poluição e assoreiam os recursos hídricos; fornecem sombra, mantendo a estabilidade térmica da água; impedem o impacto direto da chuva no solo, minimizando processos erosivos; e servem de abrigo e alimento para grande parte da fauna aquática (Lima \& Zakia, 2000; Simões, 2001; Ferreira \& Dias, 2004).

O conhecimento da organização estrutural das populações de espécies arbustivo arbóreas, por meio de estudos fitossociológicos, é base para a definição de estratégias de manejo e conservação de remanescentes florestais (Oliveira \& Amaral, 2004; Pinto et al., 2007). Kageyama \& Gandara (2000) mencionam que, além das medidas emergenciais de preservação, deve-se ainda restaurar as áreas de preservação permanente já degradadas, priorizando aquelas que permitam a implantação de uma rede de corredores florestados, interligando estes remanescentes naturais, pois, segundo Lima \& Zakia (2000), do ponto de vista ecológico, as matas ciliares têm sido consideradas como corredores extremamente importantes para o movimento da fauna ao longo da paisagem, assim como para a dispersão vegetal. Conforme Costa et al. (2007), conhecer o comportamento das espécies frente à capacidade de colonização dos ambientes a serem recuperados, como as estratégias de dispersão de propágulos é uma importante ferramenta para potencializar a capacidade de estabelecimento e de resiliência desses ambientes, não devendo ser desconsideradas durante o planejamento das práticas de revegetação.

De acordo com Van der Pijl (1982), as espécies desenvolveram diferentes mecanismos de dispersão em direção à maior eficiência. Estas estratégias constituem diferentes síndromes, adaptadas aos agentes transportadores dos diásporos. Conforme Howe \& Smallwood (1982), o conjunto de características dos frutos como morfologia, cor e época de maturação determinam a sua síndrome de dispersão.

O processo de dispersão de sementes é crucial para a reprodução das plantas, pois a semente deve chegar a um local propício para germinar, suficientemente longe da planta-mãe, a fim de evitar a competição com ela e também de predadores de sementes e plântulas que ficam nas suas proximidades (Howe, 1993; Janzen, 1970).

A condução de estudos em fragmentos de Mata Atlântica torna-se de importância fundamental para fornecer informações que permitam contribuir com a preservação dos fragmentos ainda existentes, bem como, dar suporte para ações que visem à restauração de ambientes já degradados. Portanto, o presente trabalho teve como objetivo conhecer a estrutura fitossociológica do componente arbóreo em uma mata ciliar de um córrego que deságua no Rio Sirinhaém, Município de Sirinhaém, PE, e identificar as síndromes de dispersão das espécies arbóreas amostradas. Os resultados deste trabalho ampliarão os conhecimentos sobre a vegetação nativa do Município de Sirinhaém e oferecerão informações para subsidiar a implantação de programas que visem à restauração e preservação de matas ciliares na região.

\section{Material e métodos}

\section{Caracterização da área de estudo}

O estudo foi desenvolvido em um trecho de mata ciliar do Córrego Labareda que deságua no Rio Sirinhaém, localizado no Engenho Buranhém, pertencente à Usina Trapiche S/A, no Município de Sirinhaém, PE, sob as coordenadas $8^{\circ} 34^{\prime} 38^{\prime \prime} \mathrm{S}$ e $35^{\circ} 10^{\prime} 4,9^{\prime \prime} \mathrm{W}$. 
O fragmento estudado possui uma área total de 272 ha e está inserido em uma matriz de cana-de-açúcar. Aárea foi selecionada com base em seu estado de conservação e por possuir nascentes e córregos importantes que deságuam no Rio Sirinhaém. A área apresenta altitude média de $75 \mathrm{~m}$, sendo classificada como Floresta Ombrófila Densa de Terras Baixas (IBGE, 1992).

Segundo a classificação de Köppen, a região apresenta clima do tipo As, tropical chuvoso (Vianello \& Alves, 2000), com temperatura média anual de $25,1{ }^{\circ} \mathrm{C}$. O período chuvoso tem início no outono/inverno, nos meses de junho/julho, com término em setembro. Os dados pluviométricos (1941-2009) do Departamento Agrícola da Usina Trapiche S/A indicam que a precipitação média anual é de $2.445 \mathrm{~mm}$. Os solos predominantes na área de estudo são do tipo latossolo amarelo, argissolos amarelo, vermelho-amarelo e acinzentado, gleissolo, cambissolo e solos aluviais (Silva et al., 2001; Santos et al., 2006).

\section{Coleta e análise de dados}

A vegetação ciliar foi amostrada por meio da alocação de 40 parcelas de $10 \mathrm{~m}$ x $25 \mathrm{~m}$, (20 em cada margem), sendo 10 parcelas implementadas seguindo as margens do córrego e interdistantes $25 \mathrm{~m}$, e 10 parcelas distribuídas paralelamente as primeiras (Figura 1). As 40 unidades amostrais, resultaram em $10.000 \mathrm{~m}^{2}$ (1 ha) de área amostrada.

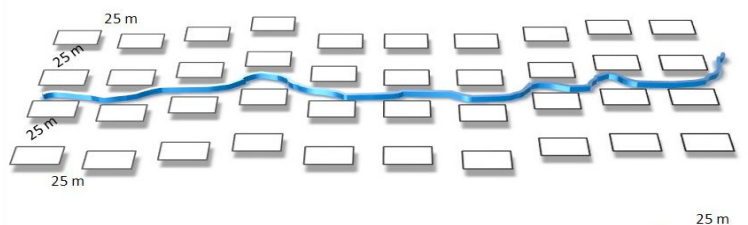

Figura 1. Croqui da distribuição das parcelas na mata ciliar, Engenho Buranhém, Usina Trapiche S/A, Sirinhaém, PE. Ilustração: Rosival Barros.

Em cada parcela, foram amostrados todos os indivíduos com circunferência à altura do peito (CAP) $\geq 15 \mathrm{~cm}$, os quais foram etiquetados e enumerados, progressivamente, com placas de PVC (5 cm x $5 \mathrm{~cm})$. Cada árvore amostrada teve o CAP mensurado com fita métrica ou trena, e a altura estimada com módulos $(1,5 \mathrm{~m}$ cada) de tesoura de alta poda. A identificação das espécies foi realizada por meio de comparações com exsicatas pertencentes ao Herbário IPA - Dárdano de Andrade Lima do Instituto Agronômico de Pernambuco, bem como por consulta a especialistas e à literatura especializada. $\mathrm{O}$ material fértil, herborizado e identificado, foi depositado no Herbário IPA e as espécies foram classificadas pelo Sistema de Cronquist (1988).

Foram analisados os parâmetros fitossociológicos de densidade absoluta (DA), densidade relativa (DR), frequência absoluta (FA), frequência relativa (FR), dominância absoluta (DoA), dominância relativa (DoR), valor de cobertura (VC) e valor de importância (VI), conforme Müeller-Dombois \& Ellemberg (1974).

A partir da caracterização taxonômica das espécies arbóreas que ocorreram no ambiente estudado, iniciouse a classificação das espécies quanto às síndromes de dispersão de sementes baseada nas características morfológicas dos diásporos descritos por Van der Pij1 (1982). Este autor classificou as espécies em três grandes categorias: zoocórica - espécie dispersa por animais; anemocórica - espécie dispersa pelo vento; autocórica - auto-dispersão. As informações sobre as síndromes de dispersão das espécies foram baseadas em observações de campo e em consultas aos estudos realizados por Talora \& Morellato (2000), Spina et al. (2001), Saravy et al. (2003), Costa et al. (2004), Gasparini Junior (2004), Neri et al. (2005), Carvalho et al. (2006), Kinoshita et al. (2006), Giehl et al. (2007), Ignácio (2007), Muniz (2008), Alencar (2009), Amaral et al. (2009), Aquino \& Barbosa (2009), Bachand et al. (2009), Emerich (2009), Silva \& Rodal (2009), Stefanello et al. (2009), Costa et al. (2010), Marangon et al. (2010), Onofre et al. (2010) e Souza (2010). Para a categorização levou-se em consideração apenas as espécies identificadas em nível de gênero e de espécie.

Foi admitido um erro amostral (E\%) de $20 \%$ e o nível de probabilidade de $95 \%$ usando a seguinte expressão (Soares et al., 2007):

$$
E \%= \pm \frac{S_{\bar{Y}} \cdot t_{\propto / 2}}{\bar{Y}}
$$

Em que: $S_{\bar{Y}}=$ erro-padrão da média; $\mathrm{t}_{\alpha / 2}=$ valor tabelado da distribuição t de Student ( $\alpha$ 5\%, n-1 gl); e $\bar{Y}=$ média do número de árvores por parcela.

\section{Resultados e discussão}

Verificou-se que as 40 unidades amostrais levantadas foram suficientes para caracterizar o fragmento de mata ciliar estudado, pois, o erro de amostragem calculado, 
levando-se em consideração o número de indivíduos por parcela, foi de $8 \%$, portanto, inferior ao erro de amostragem admissível de $20 \%$ a $95 \%$ de probabilidade.

Foram encontrados 1.307 indivíduos arbóreos na área amostrada da mata ciliar do Córrego Labareda, pertencentes a 118 espécies, 69 gêneros e 40 famílias. Ressalta-se que das 118 espécies, 22 foram identificadas apenas em nível de gênero, oito em nível de família e sete não foram identificadas (Tabela 1). A área basal estimada foi de $26,735 \mathrm{~m}^{2} \mathrm{ha}^{-1}$. Esse valor foi superior aos encontrados em formações semelhantes: Guimarães (2005) $\left(18,83 \mathrm{~m}^{2} \mathrm{ha}^{-1}\right)$; Costa Júnior (2006) $\left(23,59 \mathrm{~m}^{2}\right.$ ha-1); (Oliveira et al., 2009) $\left(24,54 \mathrm{~m}^{2} \mathrm{ha}^{-1}\right)$ e Oliveira (2011) $\left(18,8 \mathrm{~m}^{2} \mathrm{ha}^{-1}\right)$.

Tabela 1. Parâmetros fitossociológicos e síndromes de dispersão, em ordem de VI, das espécies amostradas na mata ciliar do Córrego Labareda, Sirinhaém, PE.

\begin{tabular}{|c|c|c|c|c|c|c|c|c|c|c|}
\hline Espécie & Família & SD & DA & DR & FA & FR & DoA & DoR & VI & $\mathbf{V C}$ \\
\hline Protium heptaphyllum & Burseraceae & zoo & 93 & 7,12 & 75,0 & 3,86 & 2,112 & 7,90 & 18,88 & 15,02 \\
\hline Pouteria sp. 1 & Sapotaceae & zoo & 79 & 6,04 & 80,0 & 4,12 & 0,949 & 3,55 & 13,71 & 9,59 \\
\hline Virola gardneri & Myristicaceae & zoo & 51 & 3,90 & 70,0 & 3,60 & 1,197 & 4,48 & 11,98 & 8,38 \\
\hline Simarouba amara & Simaroubaceae & zoo & 20 & 1,53 & 42,5 & 2,19 & 2,187 & 8,18 & 11,90 & 9,71 \\
\hline Cecropia pachystachya & Cecropiaceae & zoo & 51 & 3,90 & 45,0 & 2,32 & 1,05 & 3,93 & 10,15 & 7,83 \\
\hline Tovomita brevistaminea & Clusiaceae & zoo & 59 & 4,51 & 65,0 & 3,35 & 0,381 & 1,42 & 9,29 & 5,94 \\
\hline Eschweilera ovata & Lecythidaceae & auto & 44 & 3,37 & 57,5 & 2,96 & 0,772 & 2,89 & 9,21 & 6,25 \\
\hline Sclerolobium densiflorum & Caesalpiniaceae & auto & 24 & 1,84 & 40,0 & 2,06 & 1,354 & 5,06 & 8,96 & 6,90 \\
\hline Hyeronima alchorneoides & Euphorbiaceae & $\mathrm{SC}$ & 29 & 2,22 & 47,5 & 2,45 & 1,04 & 3,89 & 8,55 & 6,11 \\
\hline Pourouma acutiflora & Cecropiaceae & zoo & 23 & 1,76 & 32,5 & 1,67 & 1,25 & 4,68 & 8,11 & 6,44 \\
\hline Schefflera morototoni & Araliaceae & zoo & 18 & 1,38 & 30,0 & 1,54 & 1,345 & 5,03 & 7,95 & 6,41 \\
\hline Tapirira guianensis & Anacadiaceae & zoo & 19 & 1,45 & 35,0 & 1,8 & 1,058 & 3,96 & 7,21 & 5,41 \\
\hline Inga subnuda & Mimosaceae & zoo & 50 & 3,83 & 30,0 & 1,54 & 0,423 & 1,58 & 6,95 & 5,41 \\
\hline Cupania racemosa & Sapindaceae & zoo & 21 & 1,61 & 42,5 & 2,19 & 0,774 & 2,89 & 6,69 & 4,50 \\
\hline Ocotea glomerata & Lauraceae & $\mathrm{SC}$ & 29 & 2,22 & 40,0 & 2,06 & 0,589 & 2,20 & 6,48 & 4,42 \\
\hline Myrcia fallax & Myrtaceae & zoo & 38 & 2,91 & 52,5 & 2,70 & 0,222 & 0,83 & 6,44 & 3,74 \\
\hline Brosimum discolor & Moraceae & zoo & 33 & 2,52 & 47,5 & 2,45 & 0,349 & 1,30 & 6,27 & 3,83 \\
\hline Protium giganteum & Burseraceae & zoo & 28 & 2,14 & 50,0 & 2,57 & 0,399 & 1,49 & 6,21 & 3,63 \\
\hline Miconia prasina & Melastomataceae & zoo & 34 & 2,60 & 52,5 & 2,70 & 0,206 & 0,77 & 6,07 & 3,37 \\
\hline Anaxagorea dolichocarpa & Annonaceae & zoo & 24 & 1,84 & 37,5 & 1,93 & 0,598 & 2,24 & 6,00 & 4,07 \\
\hline Helicostylis tomentosa & Moraceae & zoo & 30 & 2,30 & 45,0 & 2,32 & 0,356 & 1,33 & 5,94 & 3,63 \\
\hline Siparuna guianensis & Monimiaceae & zoo & 35 & 2,68 & 47,5 & 2,45 & 0,194 & 0,72 & 5,85 & 3,40 \\
\hline Symphonia globulifera & Clusiaceae & zoo & 23 & 1,76 & 40,0 & 2,06 & 0,522 & 1,95 & 5,77 & 3,71 \\
\hline Ocotea cf. gardneri & Lauraceae & auto & 27 & 2,07 & 47,5 & 2,45 & 0,328 & 1,23 & 5,74 & 3,29 \\
\hline Bowdichia virgilioides & Fabaceae & ane & 15 & 1,15 & 27,5 & 1,42 & 0,614 & 2,3 & 4,86 & 3,44 \\
\hline Parkia pendula & Mimosaceae & auto & 22 & 1,68 & 35,0 & 1,80 & 0,343 & 1,28 & 4,77 & 2,97 \\
\hline Brosimum conduru & Moraceae & zoo & 18 & 1,38 & 32,5 & 1,67 & 0,455 & 1,70 & 4,75 & 3,08 \\
\hline Inga flagelliformis & Mimosaceae & zoo & 18 & 1,38 & 32,5 & 1,67 & 0,389 & 1,45 & 4,50 & 2,83 \\
\hline Thyrsodium spruceanum & Anacadiaceae & zoo & 22 & 1,68 & 32,5 & 1,67 & 0,198 & 0,74 & 4,10 & 2,42 \\
\hline Pogonophora schomburgkiana & Euphorbiaceae & auto & 19 & 1,45 & 27,5 & 1,42 & 0,284 & 1,06 & 3,93 & 2,52 \\
\hline Himatanthus phagedaenicus & Apocynaceae & ane & 16 & 1,22 & 30,0 & 1,54 & 0,111 & 0,42 & 3,19 & 1,64 \\
\hline Miconia tomentosa & Melastomataceae & zoo & 19 & 1,45 & 27,5 & 1,42 & 0,071 & 0,27 & 3,13 & 1,72 \\
\hline Sloanea sp. & Elaeocarpaceae & zoo & 5 & 0,38 & 12,5 & 0,64 & 0,554 & 2,07 & 3,10 & 2,45 \\
\hline Nectandra cuspidata & Lauraceae & zoo & 16 & 1,22 & 27,5 & 1,42 & 0,121 & 0,45 & 3,09 & 1,68 \\
\hline
\end{tabular}


Tabela 1. Continuação.

\begin{tabular}{|c|c|c|c|c|c|c|c|c|c|c|}
\hline Espécie & Família & SD & DA & DR & FA & FR & DoA & DoR & VI & VC \\
\hline Dialium guianense & Caesalpiniaceae & zoo & 7 & 0,54 & 17,5 & 0,90 & 0,383 & 1,43 & 2,87 & 1,97 \\
\hline Inga sessilis & Mimosaceae & zoo & 14 & 1,07 & 15,0 & 0,77 & 0,214 & 0,80 & 2,65 & 1,87 \\
\hline Miconia nervosa & Melastomataceae & zoo & 12 & 0,92 & 20,0 & 1,03 & 0,074 & 0,28 & 2,23 & 1,20 \\
\hline Guapira laxa & Nyctaginaceae & auto & 8 & 0,61 & 20,0 & 1,03 & 0,143 & 0,53 & 2,18 & 1,15 \\
\hline Macrosamanea pedicellaris & Mimosaceae & auto & 6 & 0,46 & 15,0 & 0,77 & 0,245 & 0,92 & 2,15 & 1,37 \\
\hline Casearia javitensis & Flacourtiaceae & zoo & 9 & 0,69 & 17,5 & 0,90 & 0,084 & 0,32 & 1,91 & 1,00 \\
\hline Pachira aquatica & Bombacaceae & auto & 7 & 0,54 & 17,5 & 0,90 & 0,102 & 0,38 & 1,82 & 0,92 \\
\hline Lecythis pisonis subsp. pisonis & Lecythidaceae & auto & 7 & 0,54 & 15,0 & 0,77 & 0,122 & 0,46 & 1,77 & 0,99 \\
\hline Coussarea sp. & Rubiaceae & $\mathrm{SC}$ & 11 & 0,84 & 15,0 & 0,77 & 0,037 & 0,14 & 1,75 & 0,98 \\
\hline Gustavia augusta & Lecythidaceae & zoo & 10 & 0,77 & 15,0 & 0,77 & 0,053 & 0,20 & 1,74 & 0,96 \\
\hline Indeterminada 6 & - & - & 5 & 0,38 & 10,0 & 0,51 & 0,191 & 0,71 & 1,61 & 1,10 \\
\hline Ficus sp. & Moraceae & zoo & 2 & 0,15 & 5,0 & 0,26 & 0,26 & 0,97 & 1,38 & 1,12 \\
\hline Guarea guidonia & Meliaceae & zoo & 6 & 0,46 & 15,0 & 0,77 & 0,037 & 0,14 & 1,37 & 0,60 \\
\hline Mabea occidentalis & Euphorbiaceae & auto & 7 & 0,54 & 5,0 & 0,26 & 0,125 & 0,47 & 1,26 & 1,00 \\
\hline Hymenaea courbaril & Caesalpiniaceae & zoo & 2 & 0,15 & 5,0 & 0,26 & 0,217 & 0,81 & 1,22 & 0,97 \\
\hline Plathymenia foliolosa & Mimosaceae & ane & 1 & 0,08 & 2,5 & 0,13 & 0,269 & 1,01 & 1,21 & 1,08 \\
\hline Pera ferruginea & Euphorbiaceae & zoo & 5 & 0,38 & 10,0 & 0,51 & 0,071 & 0,27 & 1,16 & 0,65 \\
\hline Erythroxylum citrifolium & Erythroxylaceae & zoo & 5 & 0,38 & 12,5 & 0,64 & 0,018 & 0,07 & 1,09 & 0,45 \\
\hline Quiina pernambucensis & Quiinaceae & $\mathrm{SC}$ & 6 & 0,46 & 10,0 & 0,51 & 0,018 & 0,07 & 1,04 & 0,52 \\
\hline Indeterminada 1 & - & - & 4 & 0,31 & 10,0 & 0,51 & 0,049 & 0,18 & 1,01 & 0,49 \\
\hline Eugenia sp. 1 & Myrtaceae & zoo & 4 & 0,31 & 10,0 & 0,51 & 0,047 & 0,18 & 1,00 & 0,48 \\
\hline Andira nitida & Fabaceae & zoo & 5 & 0,38 & 7,5 & 0,39 & 0,049 & 0,18 & 0,95 & 0,57 \\
\hline Meliaceae 1 & Meliaceae & - & 3 & 0,23 & 5,0 & 0,26 & 0,118 & 0,44 & 0,93 & 0,67 \\
\hline Vismia guianensis & Clusiaceae & zoo & 4 & 0,31 & 10,0 & 0,51 & 0,013 & 0,05 & 0,87 & 0,35 \\
\hline Miconia falconi & Melastomataceae & zoo & 7 & 0,54 & 5,0 & 0,26 & 0,019 & 0,07 & 0,86 & 0,61 \\
\hline Pouteria grandiflora & Sapotaceae & zoo & 4 & 0,31 & 7,5 & 0,39 & 0,039 & 0,15 & 0,84 & 0,45 \\
\hline Psychotria cf. carthagenensis & Rubiaceae & zoo & 5 & 0,38 & 7,5 & 0,39 & 0,017 & 0,06 & 0,83 & 0,45 \\
\hline Maytenus distichophylla & Celastraceae & zoo & 3 & 0,23 & 7,5 & 0,39 & 0,037 & 0,14 & 0,76 & 0,37 \\
\hline Indeterminada 4 & - & - & 4 & 0,31 & 5,0 & 0,26 & 0,05 & 0,19 & 0,75 & 0,49 \\
\hline Richeria cf. grandis & Euphorbiaceae & $\mathrm{SC}$ & 4 & 0,31 & 7,5 & 0,39 & 0,012 & 0,05 & 0,74 & 0,35 \\
\hline Ocotea limae & Lauraceae & $\mathrm{SC}$ & 4 & 0,31 & 7,5 & 0,39 & 0,011 & 0,04 & 0,73 & 0,35 \\
\hline Micropholis gardneriana & Sapotaceae & zoo & 3 & 0,23 & 7,5 & 0,39 & 0,015 & 0,06 & 0,67 & 0,29 \\
\hline Byrsonima sp. & Malpighiaceae & zoo & 3 & 0,23 & 7,5 & 0,39 & 0,013 & 0,05 & 0,66 & 0,28 \\
\hline Paypayrola blanchetiana & Violaceae & auto & 3 & 0,23 & 7,5 & 0,39 & 0,009 & 0,03 & 0,65 & 0,26 \\
\hline Chrysophyllum sp. & Sapotaceae & zoo & 2 & 0,15 & 5,0 & 0,26 & 0,054 & 0,20 & 0,61 & 0,36 \\
\hline Pouteria sp. 3 & Sapotaceae & zoo & 2 & 0,15 & 2,5 & 0,13 & 0,081 & 0,30 & 0,58 & 0,45 \\
\hline Eugenia sp. 2 & Myrtaceae & zoo & 2 & 0,15 & 5,0 & 0,26 & 0,033 & 0,12 & 0,53 & 0,28 \\
\hline Eugenia sp. 4 & Myrtaceae & zoo & 2 & 0,15 & 5,0 & 0,26 & 0,028 & 0,11 & 0,52 & 0,26 \\
\hline Indeterminada 2 & - & - & 1 & 0,08 & 2,5 & 0,13 & 0,081 & 0,30 & 0,51 & 0,38 \\
\hline Licania tomentosa & Chrysobalanaceae & zoo & 2 & 0,15 & 5,0 & 0,26 & 0,028 & 0,10 & 0,51 & 0,26 \\
\hline Rheedia brasiliensis & Clusiaceae & zoo & 2 & 0,15 & 5,0 & 0,26 & 0,023 & 0,09 & 0,50 & 0,24 \\
\hline Myrcia sp. 1 & Myrtaceae & zoo & 2 & 0,15 & 5,0 & 0,26 & 0,010 & 0,04 & 0,45 & 0,19 \\
\hline Tabernaemontana sp. & Apocynaceae & $\mathrm{SC}$ & 2 & 0,15 & 5,0 & 0,26 & 0,008 & 0,03 & 0,44 & 0,18 \\
\hline
\end{tabular}


Tabela 1. Continuação.

\begin{tabular}{|c|c|c|c|c|c|c|c|c|c|c|}
\hline Espécie & Família & SD & DA & DR & FA & FR & DoA & DoR & VI & VC \\
\hline Henrietta sucosa & Melastomataceae & zoo & 2 & 0,15 & 5,0 & 0,26 & 0,006 & 0,02 & 0,43 & 0,18 \\
\hline Brosimum gaudichaudii & Moraceae & zoo & 2 & 0,15 & 5,0 & 0,26 & 0,005 & 0,02 & 0,43 & 0,17 \\
\hline Genipa americana & Rubiaceae & auto & 2 & 0,15 & 5,0 & 0,26 & 0,005 & 0,02 & 0,43 & 0,17 \\
\hline Byrsonima sericea & Malpighiaceae & zoo & 1 & 0,08 & 2,5 & 0,13 & 0,056 & 0,21 & 0,42 & 0,29 \\
\hline Indeterminada 7 & - & - & 2 & 0,15 & 2,5 & 0,13 & 0,027 & 0,10 & 0,38 & 0,26 \\
\hline Sorocea hilarii & Moraceae & zoo & 2 & 0,15 & 2,5 & 0,13 & 0,018 & 0,07 & 0,35 & 0,22 \\
\hline Brosimum guianense & Moraceae & zoo & 1 & 0,08 & 2,5 & 0,13 & 0,032 & 0,12 & 0,32 & 0,19 \\
\hline Eugenia sp. 3 & Myrtaceae & zoo & 1 & 0,08 & 2,5 & 0,13 & 0,030 & 0,11 & 0,32 & 0,19 \\
\hline Inga laurina & Mimosaceae & zoo & 2 & 0,15 & 2,5 & 0,13 & 0,009 & 0,03 & 0,31 & 0,19 \\
\hline Ocotea sp. & Lauraceae & auto & 1 & 0,08 & 2,5 & 0,13 & 0,020 & 0,07 & 0,28 & 0,15 \\
\hline Chrysobalanaceae 1 & Chrysobalanaceae & - & 1 & 0,08 & 2,5 & 0,13 & 0,018 & 0,07 & 0,27 & 0,14 \\
\hline Lauraceae 1 & Lauraceae & - & 1 & 0,08 & 2,5 & 0,13 & 0,017 & 0,06 & 0,27 & 0,14 \\
\hline Indeterminada 3 & - & - & 1 & 0,08 & 2,5 & 0,13 & 0,014 & 0,05 & 0,26 & 0,13 \\
\hline Guatteria schomburgkiana & Annonaceae & zoo & 1 & 0,08 & 2,5 & 0,13 & 0,015 & 0,06 & 0,26 & 0,13 \\
\hline Pouteria sp. 2 & Sapotaceae & zoo & 1 & 0,08 & 2,5 & 0,13 & 0,013 & 0,05 & 0,26 & 0,13 \\
\hline Guatteria sp. & Annonaceae & zoo & 1 & 0,08 & 2,5 & 0,13 & 0,009 & 0,03 & 0,24 & 0,11 \\
\hline Tetragastris sp. & Burseraceae & $\mathrm{SC}$ & 1 & 0,08 & 2,5 & 0,13 & 0,009 & 0,03 & 0,24 & 0,11 \\
\hline Lauraceae 2 & Lauraceae & - & 1 & 0,08 & 2,5 & 0,13 & 0,005 & 0,02 & 0,23 & 0,10 \\
\hline Guarea macrophylla & Meliaceae & zoo & 1 & 0,08 & 2,5 & 0,13 & 0,007 & 0,03 & 0,23 & 0,10 \\
\hline Matayba cf. guianensis & Sapindaceae & zoo & 1 & 0,08 & 2,5 & 0,13 & 0,006 & 0,02 & 0,23 & 0,10 \\
\hline Licania sp. & Chrysobalanaceae & zoo & 1 & 0,08 & 2,5 & 0,13 & 0,003 & 0,01 & 0,22 & 0,09 \\
\hline Rheedia gardneriana & Clusiaceae & zoo & 1 & 0,08 & 2,5 & 0,13 & 0,005 & 0,02 & 0,22 & 0,10 \\
\hline Euphorbiaceae 1 & Euphorbiaceae & - & 1 & 0,08 & 2,5 & 0,13 & 0,005 & 0,02 & 0,22 & 0,10 \\
\hline Dipteryx odorata & Fabaceae & ane & 1 & 0,08 & 2,5 & 0,13 & 0,003 & 0,01 & 0,22 & 0,09 \\
\hline Carpotroche brasiliensis & Flacourtiaceae & $\mathrm{SC}$ & 1 & 0,08 & 2,5 & 0,13 & 0,005 & 0,02 & 0,22 & 0,10 \\
\hline Malpighiaceae 1 & Malpighiaceae & - & 1 & 0,08 & 2,5 & 0,13 & 0,003 & 0,01 & 0,22 & 0,09 \\
\hline Miconia hypoleuca & Melastomataceae & zoo & 1 & 0,08 & 2,5 & 0,13 & 0,004 & 0,02 & 0,22 & 0,09 \\
\hline Myrcia sp. 2 & Myrtaceae & zoo & 1 & 0,08 & 2,5 & 0,13 & 0,005 & 0,02 & 0,22 & 0,09 \\
\hline Myrtaceae 1 & Myrtaceae & - & 1 & 0,08 & 2,5 & 0,13 & 0,005 & 0,02 & 0,22 & 0,09 \\
\hline Guapira cf. nitida & Nyctaginaceae & zoo & 1 & 0,08 & 2,5 & 0,13 & 0,003 & 0,01 & 0,22 & 0,09 \\
\hline Rubiaceae 1 & Rubiaceae & - & 1 & 0,08 & 2,5 & 0,13 & 0,005 & 0,02 & 0,22 & 0,10 \\
\hline Pradosia sp. 1 & Sapotaceae & zoo & 1 & 0,08 & 2,5 & 0,13 & 0,003 & 0,01 & 0,22 & 0,09 \\
\hline Pradosia sp. 2 & Sapotaceae & zoo & 1 & 0,08 & 2,5 & 0,13 & 0,005 & 0,02 & 0,22 & 0,09 \\
\hline Indeterminada 5 & - & - & 1 & 0,08 & 2,5 & 0,13 & 0,003 & 0,01 & 0,21 & 0,09 \\
\hline Protium aracouchini & Burseraceae & zoo & 1 & 0,08 & 2,5 & 0,13 & 0,002 & 0,01 & 0,21 & 0,08 \\
\hline Sloanea guianensis & Elaeocarpaceae & zoo & 1 & 0,08 & 2,5 & 0,13 & 0,002 & 0,01 & 0,21 & 0,08 \\
\hline Swartzia pickelli & Fabaceae & auto & 1 & 0,08 & 2,5 & 0,13 & 0,002 & 0,01 & 0,21 & 0,08 \\
\hline Myrcia cf. floribunda & Myrtaceae & zoo & 1 & 0,08 & 2,5 & 0,13 & 0,003 & 0,01 & 0,21 & 0,09 \\
\hline Guapira opposita & Nyctaginaceae & zoo & 1 & 0,08 & 2,5 & 0,13 & 0,003 & 0,01 & 0,21 & 0,09 \\
\hline Alseis pickelli & Rubiaceae & ane & 1 & 0,08 & 2,5 & 0,13 & 0,003 & 0,01 & 0,21 & 0,09 \\
\hline Total & & & 1307 & 100 & 1942,5 & 100 & 26,735 & 100 & 300 & 200 \\
\hline
\end{tabular}

DA - Densidade absoluta (ind. ha ${ }^{-1}$ ); DR - Densidade relativa (\%); FA - Frequência absoluta (\%); FR - Frequência relativa (\%); DoA - Dominância absoluta $\left(\mathrm{m}^{2}\right.$ ha-1); DoR - Dominância relativa; VC - Valor de cobertura; VI - Valor de importância; SD - Síndrome de dispersão; zoo - zoocórica; ane - anemocórica; auto - autocórica; SC - Sem caracterização. 
As espécies que se destacaram em número de indivíduos, ordenadas de forma decrescente, foram: Protium heptaphyllum (Aubl.) Marchand (93), Pouteria sp. 1 (79), Tovomita brevistaminea Engl. (59), Cecropia pachystachya Trécul e Virola gardneri (A. DC.) Warb. (51 cada), Inga subnuda Salzm. ex Benth. (50), Eschweilera ovata (Cambess.) Miers (44), Myrcia fallax (Rich.) DC. (38), Siparuna guianensis Aubl. (35) e Miconia prasina (Sw.) DC. (34). Essas espécies perfizeram $37,46 \%$ do total de indivíduos amostrados. Semelhante ao relatado por Costa Júnior et al. (2008), Eschweilera ovata aparece entre as dez mais representativas em número de indivíduos, em área de Floresta Ombrófila Densa de Terras Baixas na Mata Sul de Pernambuco.

Foi observado que $30,5 \%$ das espécies foram representadas por apenas um indivíduo. Segundo Battilani et al. (2005), é muito comum em estudos fitossociológicos a ocorrência de um número elevado de espécies representadas por um ou poucos indivíduos dentro da área amostral. Essas espécies consideradas "localmente raras" (Oliveira et al., 2008) merecem atenção do ponto de vista conservacionista. $\mathrm{Na}$ área estudada, uma das espécies que merece destaque por apresentar apenas um indivíduo é a Swartzia pickelli Killip ex Ducke, já que a mesma está na lista oficial das espécies da flora brasileira ameaçadas de extinção (Brasil, 2008), portanto, sua presença é mais um indicativo da necessidade de se garantir a preservação da área.

Verificou-se para a mata ciliar estudada a presença de espécies típicas de formações ciliares, como: Cecropia pachystachya, Guarea guidonia (L.) Sleume, Guarea macrophylla Vahl, Tapirira guianensis Aubl. e Protium heptaphyllum (Battilani et al., 2005; Teixeira \& Rodrigues, 2006; Campos et al., 2007; Souza et al., 2007; Giehl \& Jarenkow, 2008).

Pouteria sp. 1 e Protium heptaphyllum são as espécies com melhor distribuição horizontal na área, com $4,12 \%$ e $3,86 \%$ para os valores de frequência relativa, respectivamente. Essas duas espécies ocorreram em mais de $75 \%$ das parcelas amostradas e apresentam o maior número de indivíduos por área. Segundo Matos \& Amaral (1999), as espécies que estão bem distribuídas ao longo da área possivelmente se tornam menos susceptíveis ao esgotamento genético.

Analisando as espécies figuradas com o maior VI, destacaram-se Protium heptaphyllum, Pouteria sp.1,
Virola gardneri, Simarouba amara Aubl., Cecropia pachystachya, Tovomita brevistaminea, Eschweilera ovata, Sclerolobium densiflorum Benth., Hyeronima alchorneoides Allemão e Pourouma acutiflora Trécul (Figura 2). O destacado valor de importância apresentado por Protium heptaphyllum, Virola gardneri, Cecropia pachystachya e Pourouma acutiflora está relacionado à sua elevada densidade relativa e dominância. Pouteria sp.1, Tovomita brevistaminea e Eschweilera ovata apresentam elevado número de indivíduos. Já as espécies Simarouba amara, Sclerolobium densiflorum e Hyeronima alchorneoides apresentaram elevado valor de área basal, além de estarem bem distribuídas na área estudada. Nas dez espécies com os maiores VI, estão concentrados $36,91 \%$ dos indivíduos amostrados, evidenciando a importância das mesmas na área de estudo.

Esses resultados corroboram parcialmente com os de Teixeira (2009), que destacou, entre as espécies de maior VI, Simarouba amara e Eschweilera ovata, em estudo realizado em Tamandaré, litoral sul de Pernambuco. Ferreira et al. (2007), estudando a mata ciliar do Açude do Meio, Reserva Ecológica de Dois Irmãos em Recife, PE, registrou a espécie Protium heptaphyllum como a segunda mais importante em termos de VI.

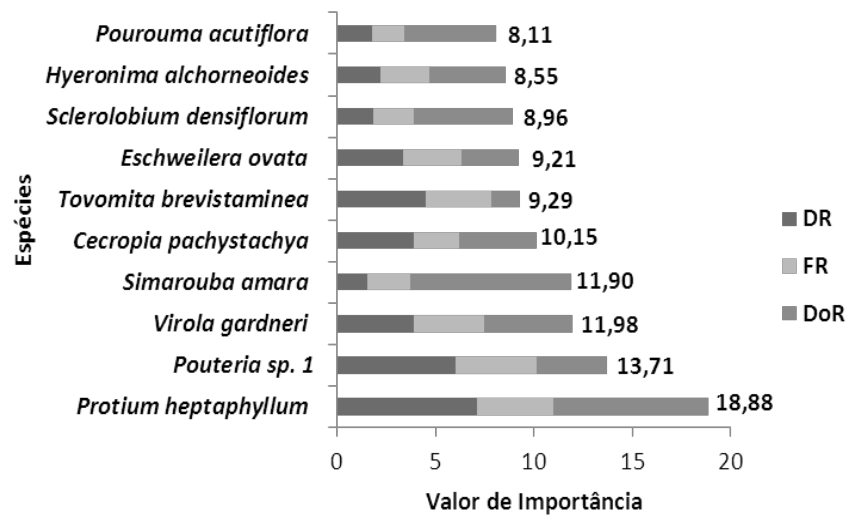

Figura 2. Dez espécies com os maiores Valores de Importância (VI), amostradas na mata ciliar do Córrego Labareda, Sirinhaém, PE. Em que: DR - Densidade relativa (\%); FR Frequência relativa (\%); DoR - Dominância relativa.

Em relação ao Valor de Cobertura (VC), as espécies Protium heptaphyllum, Simarouba amara, Pouteria sp. 1, Virola gardneri e Cecropia pachystachya apresentaram o maior número de indivíduos e as maiores dimensões. No caso da Simarouba amara, o maior valor de cobertura foi definido, principalmente 
pela dominância. Nas demais espécies foi observado que o número de indivíduos (densidade) contribuiu significativamente para a determinação do VC.

Quanto às síndromes de dispersão, dentre as 103 espécies estudadas (espécies identificadas em nível de gênero e espécie), zoocoria foi a mais frequente, representada por $72,8 \%$ do total, seguida por autocoria, $13,6 \%$ e anemocoria, 4,8\%. As espécies identificadas em nível de família ou não identificadas receberam a denominação de sem caracterização, bem como, aquelas em que não foi possível determinar a sua síndrome $(8,8 \%)$. Essas proporções se aproximam daquelas obtidas por Oliveira et al. (2011) em estudo realizado em um remanescente de Floresta Atlântica (Ombrófila Densa) no município de Moreno, PE, pois, das espécies categorizadas quanto às síndromes de dispersão, 72,8\% foram enquadradas como zoocóricas, $20 \%$ como autocóricas e $6 \%$ como espécies anemocóricas.

Em relação às famílias Anacardiaceae, Annonaceae, Araliaceae, Cecropiaceae, Celastraceae, Clusiaceae, Elaeocarparceae, Erythroxylaceae, Melastomataceae, Monimiaceae, Moraceae, Myristicaceae, Sapindaceae, Sapotaceae e Simaroubaceae, a síndrome de dispersão zoocórica esteve representada em 100\% das espécies.

No trabalho realizado por Silva \& Rodal (2009) sobre os padrões das síndromes de dispersão de plantas em áreas com diferentes graus de pluviosidade, os resultados apontaram que, nas áreas de estudo com precipitação anual inferior a $700 \mathrm{~mm}$ (Municípios de Floresta e Caruaru, PE - vegetação de caatinga), houve predomínio de vetores abióticos, enquanto que na Estação Ecológica do Tapacurá (Tapacurá), no Município de São Lourenço da Mata, PE (precipitação média anual de $1.299 \mathrm{~mm}$ - remanescente de Mata Atlântica), mais de 50\% das espécies apresentaram vetores bióticos. Howe \& Smallwood (1982) relatam que existe uma redução no percentual de plantas dispersas por animais das florestas úmidas para as secas. De acordo com Stefanello et al. (2009), ambientes florestais, estruturalmente mais complexos com menor circulação do vento e menor incidência luminosa, requerem estratégias de dispersão mais direcionadas e previsíveis como a zoocoria.

Conforme Colonetti et al. (2009), a zoocoria é um evento característico nas formações florestais da Floresta Ombrofila Densa, principalmente daquelas em estágios sucessionais mais avançados. Segundo Howe \& Smallwood (1982), nas florestas tropicais, cerca de 50\% e 75\% das árvores produzem frutos carnosos e atrativos, os quais são dispersos por pássaros e mamíferos. Para Johnson et al. (1999), não só as plantas zoocóricas são favorecidas na comunidade, mas também, os animais que delas se alimentam, influenciando desta forma a distribuição de espécies frugívoras numa comunidade.

Existe a necessidade da realização de estudos complementares em outros trechos de matas ciliares, com as mesmas características da área estudada para possibilitar comparações quanto à estrutura da vegetação, uma vez que este estudo mostra os primeiros resultados de estudos com a vegetação ciliar na região.

\section{Conclusões}

Protium heptaphyllum e Pouteria sp.1 foram as espécies mais abundantes. Em termos de Valor de Importanância (VI), as espécies Protium heptaphyllum, Pouteria sp.1 e Virola gardneri estão entre as mais importantes ecologicamente. A síndrome de dispersão mais encontrada foi a zoocoria, representada por $72,8 \%$ das espécies, seguida pela autocoria, $13,6 \%$ e a anemocoria foi observada em apenas $4,8 \%$ das espécies da mata ciliar estudada.

\section{Agradecimentos}

Ao Conselho Nacional de Desenvolvimento Científico e Tecnológico (CNPq), pela concessão de bolsa; ao Programa de Pós-graduação em Ciências Florestais da Universidade Federal Rural de Pernambuco e à Usina Trapiche S/A, pelo apoio logístico e permissão para execução do trabalho.

\section{Referências}

ALENCAR, A. L. Regeneração natural de espécies arbóreas de floresta ombrófila densa em sub-bosque de Eucalyptus saligna Smith. e Pinus caribaea Morelat var. caribaea e estudo alelopático na zona da mata sul de Pernambuco. 2009. $110 \mathrm{f}$. Dissertação (Mestrado em Botânica) - Universidade Federal Rural de Pernambuco, Recife.

AMARAL, D. D.; VIEIRA, I. C. G.; ALMEIDA, S. S.; SALOMÃO, R. P.; SILVA, A. S. L.; JARDIM, M. A. G. Checklist da flora arbórea de remanescentes florestais da região metropolitana de Belém e valor histórico dos fragmentos, Pará, Brasil. Boletim do Museu Paraense Emílio Goeldi, Belém, v. 4, n. 3, p. 231-289, 2009.

AQUINO, C.; BARBOSA, L. M. Classes sucessionais e síndromes de dispersão de espécies arbóreas e arbustivas existentes em vegetação ciliar remanescente (Conchal, SP), como subsídio para 
avaliar o potencial do fragmento como fonte de propágulos para enriquecimento de áreas revegetadas no rio Mogi-Guaçu, SP. Revista Árvore, Viçosa, MG, v. 33, n. 2, p. 349-358, 2009.

BACHAND, M.; TRUDEL, O. C.; ANSSEAU, C.; ALMEIDACORTEZ, J. Dieta de Tapirus terrestris Linnaeus em um fragmento de Mata Atlântica do Nordeste do Brasil. Revista Brasileira de Biociências, Porto Alegre, v. 7, n. 2, p. 188-194, 2009.

BATTILANI, J. L.; SCREMIN-DIAS, E.; SOUZA, A. L. T. Fitossociologia de um trecho da mata ciliar do rio da Prata, Jardim, MS, Brasil. Acta Botanica Brasilica, São Paulo, v. 19, n. 3, p. 597-608, 2005.

BRASIL. Ministério do Meio Ambiente. Instrução normativa n. 6, de 23 de setembro de 2008. Lista oficial das espécies da flora ameaçadas de extinção. Brasília, DF, 2008. Disponível em: $<$ http://portal.saude.gov.br/portal/arquivos/pdf/MMA_IN_N_6. pdf>. Acesso em: 23 jan. 2012.

CÂMARA, I. G. Brief history of conservation in the Atlantic forest. In: GALINDO-LEAL, C.; CÂMARA, I. G. (Ed.). The Atlantic Forest of South America. Washington: Center for Applied Biodiversity Science, 2003. p. 31- 42.

CAMPOS, A. C. A. L.; SANTOS, A. C. P.; VAN DEN BERG, E.; QUINELATO, M.; CERQUEIRA, F. M. Levantamento florístico e fitossociológico de mata ciliar do Rio da Mortes em São João del-Rei, Minas Gerais. Revista Brasileira de Biociências, Porto Alegre, v. 5, p. 1177-1179, 2007. Suplemento 2.

CARVALHO, F. A.; NASCIMENTO, M. T.; BRAGA, J. M. A. Composição e riqueza florística do componente arbóreo da Floresta Atlântica submontana na região de Imbaú, Município de Silva Jardim, RJ. Revista Acta Botânica Brasílica, São Paulo, v. 20, n. 3, p. 727-740, 2006.

CARVALHO, W. A. C.; OLIVEIRA FILHO, A. T.; FONTES, M. A. L.; CURI, N. Variação espacial da estrutura da comunidade arbórea de um fragmento de floresta semidecídua em Piedade do Rio Grande, MG, Brasil. Revista Brasileira de Botânica, São Paulo, v. 30, n. 2, p. 315-335, 2007.

COLONETTI, S.; CITADINI-ZANETTE, V.; MARTINS, R.; SANTOS, R.; ROCHA, E.; JARENKOW, J. A. Florística e estrutura fitossociológica em floresta ombrófila densa submontana na barragem do rio São Bento, Siderópolis, Estado de Santa Catarina. Acta Scientiarum. Biological Sciences, Maringá, v. 31, n. 4, p. 397-405, 2009.

COSTA, I. R.; ARAUJO, F. S.; LIMA-VERDE, L. W. Flora e aspectos auto-ecológicos de um encrave de cerrado na chapada do Araripe, Nordeste do Brasil. Acta Botanica Brasilica, São Paulo, v. 18 , n. 4, p. 759-770, 2004.

COSTA JÚNIOR, R. F. Caracterização estrutural de um remanescente de Mata Atlântica do município de CatendePE. 2006. 52 f. Dissertação (Mestrado em Ciências Florestais) Universidade Federal Rural de Pernambuco, Recife.

COSTA JÚNIOR, R. F.; FERREIRA, R. L. C.; RODAL, M. J. N.; FELICIANO, A. L. P.; MARANGON, L. C.; SILVA, W. C. Estrutura fitossociológica do componente arbóreo de um fragmento de floresta ombrófila densa na Mata Sul de Pernambuco, Nordeste do Brasil. Ciência Florestal, Santa Maria, v. 18, n. 2, p. 173-183, 2008.

COSTA, M. P.; NAPPO, M. E.; CAÇADOR, F. R. D.; BARROS, H. H. D. Avaliação do processo de reabilitação de um trecho de floresta ciliar na bacia do Rio Itapemirim-ES. Revista Árvore, Viçosa, MG, v. 34, n. 5, p. 835-851, 2010.

COSTA, M. P.; NAPPO, M. E.; CAÇADOR, F. R. D.; BARROS, H. H. D. Interpretação de guildas ecológicas das espécies arbóreas utilizadas na vegetação de mata ciliar em Alegre - ES. In: CONGRESSO DE ECOLOGIA DO BRASIL, 8., 2007, Caxambu. Anais... Caxambu: Sociedade de Ecologia do Brasil, 2007. p. 1-2.

CRONQUIST, A. The evolution and classification of flowering plants. New York: The New York Botanical Garden, 1988. 555 p.

EMERICH, K. H. Composição florística e relação entre variáveis ambientais e a estrutura da comunidade arbórea de fragmento florestal ciliar do rio Turvo, município de Turvo, Santa Catarina. 2009. 81 f. Dissertação (Mestrado em Ciências Ambientais) - Universidade do Extremo Sul Catarinense, Criciúma.

FERREIRA, D. A. C.; DIAS, H. C. T. Situação atual da mata ciliar do Ribeirão São Bartolomeu em Viçosa, MG. Revista Árvore, Viçosa, MG, v. 28, n. 4, p. 617-623, 2004.

FERREIRA, R. L. C.; MARANGON, L. C.; SILVA, J. A. A.; ROCHA, M. S.; ALVES JÚNIOR, F. T.; APARÍCIO, P. S.

Estrutura fitossociológica da Mata Ciliar do Açude do Meio, Reserva Ecológica de Dois Irmãos, Recife-PE. Magistra, Cruz das Almas, v. 19, n. 1, p. 31-39, 2007.

GALINDO-LEAL, C.; CÂMARA, I. G. Status do hotspot Mata Atlântica: uma síntese. In: GALINDO-LEAL, C.; CÂMARA, I. G. (Ed.). Mata Atlântica: biodiversidade, ameaças e perspectivas. São Paulo: Fundação SOS Mata Atlântica, 2005. p. 3 -11.

GASPARINI JUNIOR, A. J. Estrutura e dinâmica de um fragmento de floresta estacional semidecidual no campus da Universidade Federal de Viçosa - Viçosa (MG). 2004. $54 \mathrm{f}$.

Tese (Doutorado em Botânica) - Universidade Federal de Viçosa, Viçosa, MG.

GIEHL, E. L. H.; ATHAYDE, E. A.; BUDKE, J. C.; GESING, J. P. A.; EINSIGER, S. M.; CANTO-DOROW, T. S. Espectro e distribuição vertical das estratégias de dispersão de diásporos do componente arbóreo em uma floresta estacional no sul do Brasil. Acta Botanica Brasilica, São Paulo, v. 21, n. 1, p. 137-145, 2007.

GIEHL, E. L. H; JARENKOW, J. A. Gradiente estrutural no componente arbóreo e relação com inundações em uma floresta ribeirinha, rio Uruguai, sul do Brasil. Acta Botanica Brasilica, São Paulo, v. 22, n. 3, p. 741-753, 2008.

GUIMARÃES, F. J. P. Avaliação da estrutura de um fragmento florestal no município de Catende-PE. 2005. 67 f. Dissertação (Mestrado em Ciências Florestais) - Universidade Federal Rural de Pernambuco, Recife.

HOWE, H. F. Aspects of variation in a neotropical seed dispersal system. Vegetatio, Ames, v. 107, n. 108, p. 149-162, 1993. 
HOWE, H. F.; SMALLWOOD, J. Ecology of seed dispersal. Annual Review Ecolology and Systematics, v. 13, p. 201-228, 1982.

IBGE. Manual técnico da vegetação brasileira. Rio de Janeiro: CDDI-IBGE, 1992. 92 p. (Série manuais técnicos em geociências, 1).

IGNÁCIO, M. Estrutura, diversidade e dispersão em floresta ombrófila densa no sul da Bahia, Brasil. 2007. 81 f. Dissertação (Mestrado em Botânica) - Universidade Federal de Viçosa, Viçosa, MG.

JANZEN, D. H. Herbivores and the number of tree species in tropical forests. American Naturalist, v. 104, p. 501-528, 1970.

JOHNSON, M. A.; SARAIVA, P. M.; COELHO, D. The role of gallery forests in the distribution of Cerrado mammals. Revista Brasileira de Biologia, São Carlos, v. 59, n. 3, p. 421-427, 1999.

KAGEYAMA, P. Y. ; GANDARA, F. B. Recuperação de áreas ciliares. In: RODRIGUES, R. R.; LEITÃO-FILHO, H. F. Matas ciliares: uma abordagem multidisciplinar. São Paulo: EDUSP, 2000. $241 \mathrm{p}$.

KINOSHITA, L. S.; TORRES, R. B.; FORNI-MARTINS, E. R.; SPINELLI, T.; AHN, Y. J.; CONSTÂNCIO, S. S. Composição florística e síndromes de polinização e dispersão da mata do Sitio São Francisco, Campinas, SP, Brasil. Acta Botanica Brasílica, São Paulo, v. 20, n. 2, p. 313-327, 2006.

LIMA, W. P.; ZAKIA, M. J. B. Hidrologia de matas ciliares. In: RODRIGUES, R. R.; LEITÃO-FILHO, H. F. (Ed.). Matas ciliares: conservação e recuperação. 2. ed. São Paulo: EDUSP/ FAPESP, 2000. p. 33-44.

MARANGON, G. P.; CRUZ, A. F.; BARBOSA, W. B.; LOUREIRO, G. H.; HOLANDA, A. C. Dispersão de sementes de uma comunidade arbórea em um remanescente de mata atlântica, município de Bonito, PE. Revista Verde, Mossoró, v. 5, n. 5, p. 80-87, 2010.

MATOS, F. D. A.; AMARAL, I. L. Análise ecológica de um hectare em floresta ombrófila densa de terra firme, estrada da várzea, Amazonas, Brasil. Acta Amazonica, Manaus, v. 29, n. 3 , p. 365-379, 1999.

MORENO, M. R.; NASCIMENTO, M. T.; KURTZ, B. C. Estrutura e composição florística do estrato arbóreo em duas zonas altitudinais na mata atlântica de encosta da região do Imbé, RJ.

Acta Botanica Brasilica, São Paulo, v. 17, n. 3, p. 371-386, 2003.

MÜLLER-DOMBOIS, D.; ELLEMBERG, H. Aims and methods of vegetation ecology. New York: John Wiley \& Sons, 1974. $547 \mathrm{p}$.

MUNIZ, F. H. Padrões de floração e frutificação de árvores da Amazônia Maranhense. Acta Amazonica, Manaus, v. 38, n. 4, p. 617-626, 2008.

NERI, A. V.; CAMPOS, E. P.; DUARTE, T. G.; MEIRA NETO, J. A. A.; SILVA, A. F.; VALENTE, G. E. Regeneração de espécies nativas lenhosas sob plantio de Eucalyptus em área de Cerrado na Floresta Nacional de Paraopeba, MG, Brasil. Acta Botanica Brasilica, São Paulo, v. 19, n. 2, p. 369-376, 2005.

OLIVEIRA, A. N.; AMARAL, I. L. Florística e fitossociologia de uma floresta de vertente na Amazônia Central, Amazonas, Brasil. Acta Amazonica, Manaus, v. 34, n. 1, p. 21-34, 2004.

OLIVEIRA, A. N.; AMARAL, I. L.; RAMOS, M. B. P.; NOBRE,
A. D.; COUTO, L. B.; SAHDO, R. M. Composição e diversidade florístico-estrutural de um hectare de floresta densa de terra firme na Amazônia Central, Amazonas, Brasil. Acta Amazonica, Manaus, v. 38, n. 4, p. 627-642, 2008.

OLIVEIRA, E. B.; MARANGON, L. C.; FELICIANO, A. L. P.; FERREIRA, R. L. C.; PIETRO, L. R. Estrutura fitossociológica de um fragmento de mata ciliar, Rio Capibaribe Mirim Aliança, Pernambuco. Revista Brasileira de Ciências Agrárias, Recife, v. 4, n. 2, p. 167-172, 2009.

OLIVEIRA, L. S. B. Estudo do componente arbóreo e efeito de borda em fragmentos de Floresta Atlântica na Bacia hidrográfica do Rio Tapacurá-PE. 2011. 92 f. Dissertação (Mestrado em Ciências Florestais) - Universidade Federal Rural de Pernambuco, Recife.

OLIVEIRA, L. S. B.; MARANGON, L. C.; FELICIANO, A. L. P.; LIMA, A. S.; CARDOSO, M. O.; SILVA, V. F. Florística, classificação sucessional e síndromes de dispersão em um remanescente de Floresta Atlântica, Moreno-PE. Revista Brasileira de Ciências Agrárias, Recife, v. 6, n. 3, p. 502-507, 2011.

ONOFRE, F. F.; ENGEL, V. L.; CASSOLA, H. Regeneração natural de espécies da Mata Atlântica em sub-bosque de Eucalyptus saligna Smith. em uma antiga unidade de produção florestal no Parque das Neblinas, Bertioga, SP. Scientia Forestalis, Piracicaba, SP, v. 38, n. 85, p. 39-52, 2010.

PINTO, S. I. C.; MARTINS, S. V.; SILVA, A. G.; BARROS, N. F.; DIAS, H. C. T.; SCOSS, L. M. Estrutura do componente arbustivo-arbóreo de dois estádios sucessionais de floresta estacional semidecidual na reserva florestal mata do paraíso, viçosa, MG, Brasil. Revista Árvore, Viçosa, MG, v. 31, n. 5, p. 823-833, 2007.

SANTOS, H. G. dos; JACOMINE, P. K. T.; ANJOS, L. H. C. dos; OLIVEIRA, V. A. de; OLIVEIRA, J. B. de; COELHO, M. R.; LUMBRERAS, J. F.; CUNHA, T. J. F. (Ed.). Sistema brasileiro de classificação de solos. 2. ed. Rio de Janeiro: Embrapa Solos, 2006. 412 p.

SANTOS, R. M.; VIEIRA, F. A. Florística e estrutura da comunidade Arbórea de fragmentos de matas Ciliares dos rios São Francisco, Cochá e Carinhanha, Norte de Minas Gerais, Brasil.

Revista Científica Eletrônica de Engenharia Florestal, Garça, v. 4, n. 8, p. 1-18, 2006.

SARAVY, F. P.; FREITAS, P. J.; LAGE, M. A.; LEITE, S. J.; BRAGA, L. F.; SOUSA, M. P. Síndrome de dispersão em estratos arbóreos em um fragmento de floresta ombrófila aberta e densa em Alta Floresta - MT. Revista do Programa de Ciências AgroAmbientais, Alta Floresta, v. 2, n. 1, p. 1-12, 2003.

SILVA, F. B. R.; SANTOS, J. C. P. dos; SILVA, A. B. da; CAVALCANTI, A. C.; SILVA, F. H. B. B. da; BURGOS, N.; PARAHYBA, R. da B. V.; OLIVEIRA NETO, M. B. de; SOUSA NETO, N. C. de; ARAÚJO FILHO, J. C. de; LOPES, O. F.; LUZ, L. R. Q. P. da; LEITE, A. L.; SOUZA, L. de G. M. C.; SILVA, C. P. da; VAREJÃO-SILVA, M. A.; BARROS, A. H. C. Zoneamento agroecológico do Estado de Pernambuco. Recife: Embrapa Solos UEP Recife: Governo do Estado de Pernambuco - Secretaria de Produção Rural e Reforma Agrária. 2001.

(Embrapa Solos. Documentos, 35). 
SILVA, M. C. N. A.; RODAL, M. J. N.; Padrões das síndromes de dispersão de plantas em áreas com diferentes graus de pluviosidade, PE, Brasil. Acta Botanica Brasílica, São Paulo, v. 23, n. 4, p. 1040-1047, 2009.

SIMÕES, L. B. Integração entre um modelo de simulação hidrológica e sistema de informação geográfica na delimitação de zonas tampão ripárias. 2001. 171 f. Tese (Doutorado em Agronomia) - Universidade Estadual de São Paulo, Botucatu.

SOARES, C. P. B.; PAULA NETO, F.; SOUZA, A. L. Dendrometria e inventário florestal. Viçosa, MG: UFV, 2007. 276 p.

SOUZA, A. L.; SCHETTINO, S.; JESUS, R. M.; VALE, A. B. Dinâmica da regeneração natural em uma floresta Ombrófila Densa secundária, após corte de cipós, reserva natural da Companhia Vale do Rio Doce S.A., Estado do Espírito Santo, Brasil. Revista Árvore, Viçosa, MG, v. 26, n. 4, p. 411-419, 2002.

SOUZA, F. N.; MELO, D. D. V.; GOMES, F. P.; SCOLFORO, R. S.; MELLO, J. M. Composição florística e estrutural de fragmentos de mata ciliar na bacia do Rio São Francisco, MG. Revista Brasileira de Biociências, Porto Alegre, v. 5, p. 285-287, 2007. Suplemento 2 .

SOUZA, J. T. Chuva de sementes em área abandonada após cultivo próxima a um fragmento preservado de caatinga em Pernambuco, Brasil. 2010. 60 f. Dissertação (Mestrado em Botânica) - Universidade Federal Rural de Pernambuco, Recife.
SPINA, A. P.; FERREIRA, W. M.; LEITÃO FILHO, H. F. Floração, frutificação e síndromes de dispersão de uma comunidade de floresta de brejo na região de Campinas (SP). Revista Acta Botânica Brasílica, São Paulo, v. 15, n. 3, p. 349368, 2001.

STEFANELLO, D.; FERNANDES-BULHÃO, C.; MARTINS, S. V. Síndromes de dispersão de sementes em três trechos de vegetação ciliar (nascente, meio e foz) ao longo do Rio Pindaíba, MT. Revista Árvore, Viçosa, MG, v. 33, n. 6, p. 1051-1061, 2009.

TALORA, D. C.; MORELLATO, P. C. Fenologia de espécies arbóreas em floresta de planície litorânea do sudeste do Brasil. Revista Brasileira de Botânica, São Paulo, v. 23, n. 1, p. 13-26, 2000.

TEIXEIRA, A. P.; RODRIGUES, R. R. Análise florística e estrutural do componente arbustivo-arbóreo de uma florestal de galeria no Município de Cristais Paulistas, SP, Brasil. Acta Botanica Brasilica, São Paulo, v. 20, n. 4, p. 803-813, 2006.

TEIXEIRA, L. J. Fitossociologia e florística do componente arbóreo em topossequência na Reserva Biológica de Saltinho, Pernambuco. 2009. 79 f. Dissertação (Mestrado em Ciências Florestais) - Universidade Federal Rural de Pernambuco, Recife.

VAN DER PIJL, L. Principles of dispersal in higher plants. Berlin: Springer Verlag, 1982. 215 p.

VIANELLO, R. L.; ALVES, A. R. Meteorologia básica e aplicações. Viçosa, MG: UFV, 2000. 449 p. 
